\title{
Supershells in Metal Clusters: Self-Consistent Calculations and their Semiclassical Interpretation
}

\author{
Erik Koch \\ Max-Planck-Institut für Festkörperforschung, D-70569 Stuttgart
}

(28 September 1995)

\begin{abstract}
To understand the electronic shell- and supershell-structure in large metal clusters we have performed self-consistent calculations in the homogeneous, spherical jellium model for a variety of different materials. A scaling analysis of the results reveals a surprisingly simple dependence of the supershells on the jellium density. It is shown how this can be understood in the framework of a periodic-orbit-expansion by analytically extending the well-known semiclassical treatment of a spherical cavity to more realistic potentials.
\end{abstract}

36.40.Cg, 31.15.Ew, 31.15.Gy

cond-mat/9606023

The structure observed in the mass spectra of large, warmed metal clusters [1] 4 ] can be attributed to the properties of itinerant electrons moving in a finite volume [5.6]. The most prominent finite-size effect is the occurrence of pronounced oscillations in the density of states 17] giving rise to an oscillating part $\tilde{E}$ of the total energy, which is superimposed on the smooth Thomas-Fermi energy $\bar{E}$. With increasing cluster radius, one finds regular oscillations (shells) whose amplitude is modulated (supershells).

Two different theoretical approaches have been used to describe the electronic shell- and supershell-structure in large metal clusters. One is the self-consistent jellium model [8]. In its simplest form a cluster is described by a homogeneous sphere of given charge density, dressed with $N$ valence electrons. Treating the electrons selfconsistently using density functional theory, the only input parameter for such a calculation is the Wigner-Seitz radius $r_{s}$. Although describing the electronic structure of alkali clusters quite well, this model provides little physical insight into the mechanisms underlying the shell- and supershell oscillations. It is here that the second approach comes in. Given an effective one-particle potential one can find a semiclassical expansion of the oscillating part of the density of states in terms of classical periodic orbits [7,9]. Introducing a suitable damping factor one finds for the spherical cavity, that the oscillations in the density of states are essentially determined by the contributions of triangular and square orbits. The supershells can thus be understood as a beating pattern originating from the contributions of these orbits.

The semiclassical approach draws its power from the fact that the periodic-orbit-expansion is known analytically for the model case of the spherical cavity. However, potentials with hard walls are only a crude approximation to realistic cluster potentials, which have a soft surface. Such potentials can also be treated using semiclassical techniques 10,11. In particular ultra-soft potentials have received much attention $[12,13]$. Unfortunately in these cases the action integrals entering the semiclassical formalism have to be evaluated numerically.
In order to find out what determines the shell- and supershell-structure in metal clusters, we have carried out the following program. First we performed a series of calculations in the homogeneous, spherical jellium model for a range of different electron densities. A scaling analysis of the results suggested that changing the electron density merely introduces a phase shift in the supershell structure. It is shown how this can be understood semiclassically in terms of a leptodermous expansion, where the action integrals for the potential under consideration are expanded around a cavity. Thereby we obtain an analytical expression for the shift of the supershells. Finally it is shown that the leptodermous expansion works for realistic cluster potentials by comparing the shifts of supershells extracted from self-consistent calculations to those given by the semiclassical formulae.

As starting point of our analysis, we have performed extensive calculations in the homogeneous, spherical jellium model. We use the local density approximation in the parameterization given in Ref. 14. Electron densities range from $r_{s}=2.07 a_{0}$ for aluminum to $r_{s}=5.63 a_{0}$, corresponding to bulk cesium. Cluster sizes were chosen from $N=100$ to 6000 valence-electrons, thus including the first two nodes of the supershell oscillation for all densities considered. Typical results for $\tilde{E}(N)$ are shown in fig. 1. It can be seen that the supershells are shifted towards larger $N$ as the Wigner-Seitz radius decreases, while the positions of the shell-minima are fairly independent of $r_{s}$. To quantify the supershells we determine the envelope of $\tilde{E}(N)$ by low-pass filtering its absolute value. The position of the supershell-nodes are given by the minima in the envelope. Filtering out the shell structure of course introduces an uncertainty of the order of the distance between adjacent shell-minima. The results of our jellium calculations are listed in table 1 .

To make a quantitative comparison of the different jellium calculations, we describe the problem in terms of dimensionless quantities. In order to do so, the relevant scales of the problem have to be identified. Obviously one such scale is the Wigner-Seitz radius. In fact, we find that the amplitude of the oscillations $\tilde{E}(N)$ are 

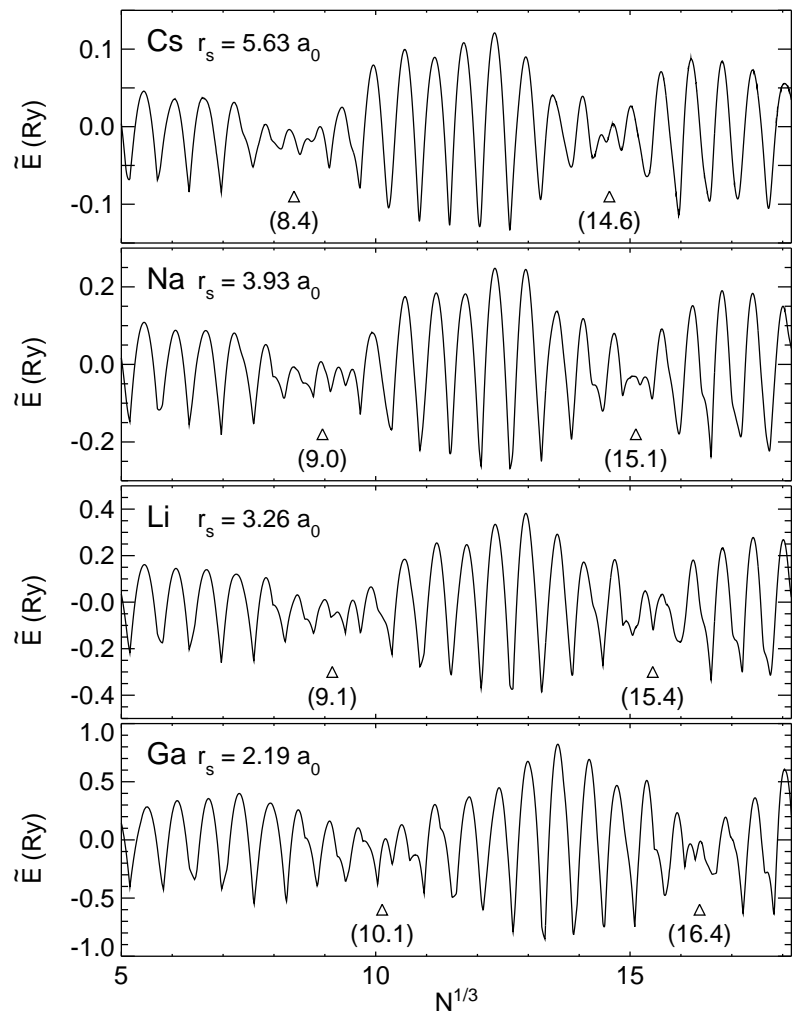

FIG. 1. Oscillating part of the total energy extracted from self-consistent calculations in the homogeneous, spherical jellium model. The positions of the super-nodes are indicated.

proportional to $1 / r_{s}^{2}$. The existence of a surface introduces an additional scale: the width $a$ of the surface region. As has been shown in [15], $a$ is fairly independent of $r_{s}$. Assuming the shift of the supershells to be a surfaceeffect, we identify $a / r_{s} \propto 1 / r_{s}$ as the relevant scaling parameter. By plotting the positions of the super-nodes as a function of $1 / r_{s}$ (cf. fig. 2) we indeed find a simple relation: The super-nodes are linearly shifted as a function of $1 / r_{s}$. In particular, the first and second super-node are shifted in parallel. Describing $\tilde{E}(N)$ semiclassically as a simple beating pattern [5], it is therefore tempting to conclude that the shift of the supershells is caused by a phase-shift in the contributions of the periodic orbits.

\begin{tabular}{lccc}
\hline \hline material & $r_{s}$ in $a_{0}$ & 1st super-node & 2nd super-node \\
\hline $\mathrm{Cs}$ & 5.63 & 8.39 & 14.59 \\
$\mathrm{Rb}$ & 5.20 & 8.43 & 14.67 \\
$\mathrm{~K}$ & 4.86 & 8.47 & 14.75 \\
$\mathrm{Na}$ & 3.93 & 8.95 & 15.11 \\
$\mathrm{Li}$ & 3.26 & 9.15 & 15.45 \\
$\mathrm{Tl}$ & 2.48 & 9.79 & 16.01 \\
$\mathrm{In}$ & 2.41 & 9.85 & 16.11 \\
$\mathrm{Ga}$ & 2.19 & 10.13 & 16.37 \\
$\mathrm{Al}$ & 2.07 & 10.20 & 16.49 \\
\hline \hline
\end{tabular}

TABLE I. Position of super-nodes (given as $N^{1 / 3}$ ) for different jellium-densities.
To check this conjecture, we derive an explicit periodicorbit-expansion for the oscillating part $\tilde{E}(N)$ of the total energy. Our approach is based on the fact that $\tilde{E}$ can, to first order, be extracted from the spectrum of smooth potentials that fit the self-consistent results [16]. We start from the observation 17 that in the semiclassical approximation the density of states naturally separates into two contributions: the smooth Thomas-Fermi term $\bar{\rho}$ and an oscillating contribution $\tilde{\rho}$. The latter term describes the quantum corrections to Thomas-Fermi theory and is given by an expansion over all classical periodic orbits which exist in the potential under consideration. For a spherically symmetric potential with exactly two classical turning points the periodic orbits can be uniquely labeled by two positive integers: the number of times $\lambda$ it turns around the origin, and the number $\nu$ of vertices it has. Denoting the classical action along such a periodic orbit by $S_{(\lambda, \nu)}$, one finds an expression of the form [7]:

$$
\tilde{\rho}(E) d E=\sum_{(\lambda, \nu)} A_{(\lambda, \nu)} \cos \left(S_{(\lambda, \nu)} / \hbar-\varphi_{(\lambda, \nu)}\right) d E
$$

where $\varphi_{(\lambda, \nu)}$ is the so called Maslov-phase. Unfortunately the expansion (11) converges quite slowly. This is obvious, since it is supposed to approximate the density of states, which is a sum of $\delta$-functions. Therefore one usually introduces some damping as to broaden the eigenstates and make the expansion (1) converge more rapidly. But we are actually not interested in the density of states, rather in $\tilde{E}(N)$. In the limit of large $N$ (which corresponds to the semiclassical limit) the oscillating part of the total energy is given by

$$
\tilde{E}(N)=-\int_{0}^{\bar{E}_{F}(N)} d E \int_{0}^{E} d E^{\prime} \tilde{\rho}\left(N ; E^{\prime}\right) .
$$

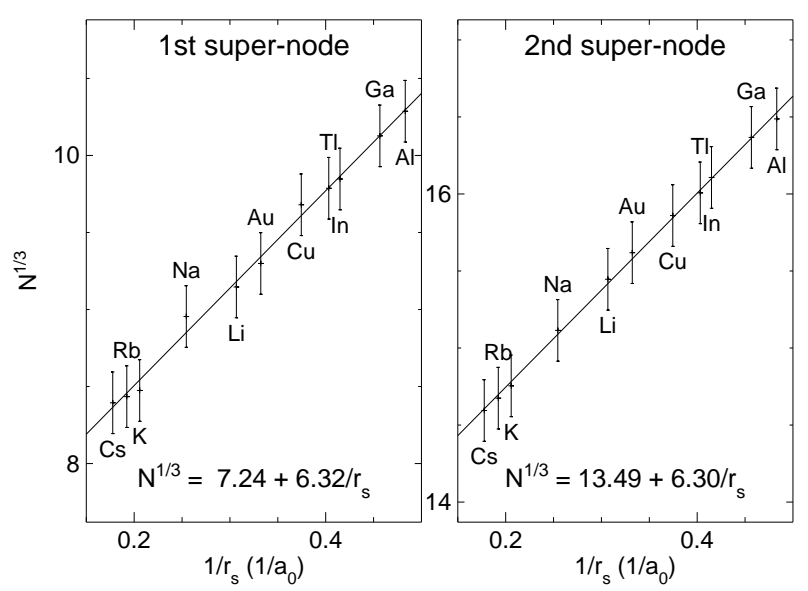

FIG. 2. Position of the first and second super-node as a function of $1 / r_{s}$. The error bars are due to the uncertainty in locating the super-nodes as minima of the envelope of $\tilde{E}(N)$. The solid line gives a linear fit to the data, the parameters of which are given in the plot. 
Here $\bar{E}_{F}(N)$ is the Fermi energy in Thomas Fermi approximation. Integrating twice by parts over $\tilde{\rho}$ essentially divides the expansion parameters $A_{(\lambda, \nu)}$ in (11) by the square of the classical action along the orbit, thereby reducing the importance of the longer orbits:

$$
\tilde{E}(N)=\bar{E}_{F}^{2} \sum_{(\lambda, \nu)} \frac{4 A_{(\lambda, \nu)}}{S_{(\lambda, \nu)}^{2}} \cos \left(S_{(\lambda, \nu)} / \hbar-\varphi_{(\lambda, \nu)}\right) .
$$

Thus there is no need of introducing a damping factor to accelerate convergence. Actually, $\tilde{E}(N)$ is dominated by the contributions of the shortest plane periodic orbits, namely the triangular and the square orbit. Furthermore inspection of (3) shows that variations in the boundary conditions, which strongly shift the oscillations in $\tilde{\rho}[18]$, hardly influence $\tilde{E}$, since the changes in the density of states are compensated by those in the Fermi energy.

As an immediate application of the expansion (B), we can investigate how an increase in density for small clusters compared to the bulk affects the electronic shells and supershells. Such a lattice contraction was suggested by EXAFS analyses of small clusters 19 . Changing the density clearly will change the Fermi-energy for a given cluster. As we can see from equation (3) this will obviously change the overall amplitude of $E(N)$. But apart from that the oscillations are determined by the classical actions $S_{(\lambda, \nu)}$ along the periodic orbits. For a spherical cavity of radius $R_{0}$ we find

$$
S_{(\lambda, \nu)} / \hbar=2 \nu \sin (\pi \lambda / \nu) \bar{k}_{F} R_{0} ; \quad \bar{k}_{F}=\sqrt{2 m \bar{E}_{F}} / \hbar,
$$

where the product $\bar{k}_{F} R_{0}$ depends on the number $N$ of electrons inside the cavity, but is independent of the electron-density. Hence the electronic shell-structure for spherical-cavity-clusters does not depend on any lattice contraction, except for an overall change in amplitude. This result suggests that the same is true for smooth potentials, provided the lattice contraction is not too large. We have confirmed this by numerically solving the quantum mechanical problem for realistic potentials introducing contractions $\Delta R_{0}$ of up to $1 / 2 r_{s}$. Thus we can conclude that a possible lattice contraction will not noticeably affect the electronic shells and supershells.

Next we turn to the problem of understanding why the super-nodes are phase-shifted as a function of jellium density (cf. fig. 2). The most straightforward approach would be to solve the integrals, which enter equation (3), explicitly. Unfortunately, this cannot be done analytically. But in the semiclassical limit, which corresponds to $N \rightarrow \infty$, it is sufficient to know the integrals to leading order in $1 / N$. The basic idea is then to use the spherical cavity as a starting point and expand the action for more realistic potentials around this case, using the surfacewidth $a$ as small parameter (leptodermous expansion). For the classical action we then find

$$
S_{(\lambda, \nu)} / \hbar=S_{(\lambda, \nu)}^{\text {cavity }} / \hbar+\left(I_{1}+I_{2} \frac{a}{r_{s}}\right)+\mathcal{O}\left(N^{-1 / 3}\right),
$$

where the expansion parameters $I_{1}$ and $I_{2}$ are independent of cluster-size; i.e. to leading order surface softness introduces a phase-shift in the periodic-orbit-expansion (3), while the period of the oscillations is still determined by $S_{(\lambda, \nu)}^{\text {cavity }} / \hbar$. An additional phase shift arises from the difference of the Maslov phases $\varphi_{(\lambda, \nu)}$ for a soft potential or a cavity. Finally an inspection of the amplitudes in (3) shows that, to leading order in $1 / N$, they do not dependent on the shape of the potential. We thus find that replacing a cavity by a soft potential with small surfaceparameter $a$ amounts to merely shifting phases in the periodic-orbit-expansion of $\tilde{E}(N)$.

Now the question arises, whether typical cluster potentials are such that their surface-parameter is small enough for the above expansion to be valid. To judge this, we have to fit the potentials obtained from our selfconsistent calculation with some analytical model potential. Since the classical action depends only on the potential in the classically allowed region, it seems reasonable to fit only for $E<E_{F}$. There the self-consistent potential, except for possible Friedel oscillations, can be well described by a Woods-Saxon function

$$
V(r)=\frac{-V_{0}}{1+\exp \left(\frac{r-R_{0}}{a}\right)} .
$$

But fitting only for $E<E_{F}$ seems to imply an error in calculating the Maslov phases which serve to capture the influence of the classically forbidden region. From WKB quantization it can be seen that the Maslov phases for a separable system are given by the sum of the quantum-mechanical scattering-phases at the classical turning points. For Woods-Saxon potentials we can calculate these analytically. To leading order, they coincide with the Maslov phases for a square-well potential of depth $-V_{0}$ [18]. Thus, the error in the potential for $E>E_{F}$ will not enter the leptodermous expansion.

Given the potential (6), we have found analytical expressions for the parameters $I_{1}$ and $I_{2}$ in the expansion (5) of the classical action. Introducing the abbreviations

$$
P=\sqrt{\frac{E_{F}+V_{0}}{V_{0}}} \text { and } P_{L}=P \cos \left(\frac{\pi \lambda}{\nu}\right),
$$

the expansion parameters for a given periodic orbit $(\lambda, \nu)$ are

$$
I_{1}=\frac{3 \nu}{2} \sin \left(\frac{\pi \lambda}{\nu}\right)\left[\left(\frac{1}{P^{2}}-2\right) \arcsin (P)-\sqrt{\frac{1}{P^{2}}-1}\right]
$$

and

$$
\begin{aligned}
& I_{2}=4 \nu\left(\frac{9 \pi}{4}\right)^{\frac{1}{3}}\left[\frac{P_{L}}{P} \ln \left(2 P_{L}\right)-\sqrt{\frac{1-P_{L}^{2}}{P^{2}}} \arcsin \left(P_{L}\right)\right. \\
& \left.-\sin \left(\frac{\pi \lambda}{\nu}\right)\left(\ln (2 P)+\left(\frac{1}{P^{2}}-1\right)^{\frac{3}{2}} \arcsin (P)-\frac{1}{P^{2}}\right)\right] .
\end{aligned}
$$


Details of the calculation will be published elsewhere [20].

We are now in the position to find a simple estimate for the shift of the supershells. Following [5] we start from a drastically simplified version of equation (3): All periodic orbits except triangular $(1,3)$ and the square $(1,4)$ orbits are neglected. Furthermore it is assumed that the amplitudes for these orbits are equal. This leaves us with an expression of the form

$$
\tilde{E}=A\left(\cos \left(f_{1} N^{\frac{1}{3}}+\varphi_{1}\right)+\cos \left(f_{2} N^{\frac{1}{3}}+\varphi_{2}\right)\right) .
$$

Fitting the self-consistent potentials with (6), we can compare the shift observed in the jellium calculations to that determined by the leptodermous expansion using the ansatz (7). This is shown in fig. 3.

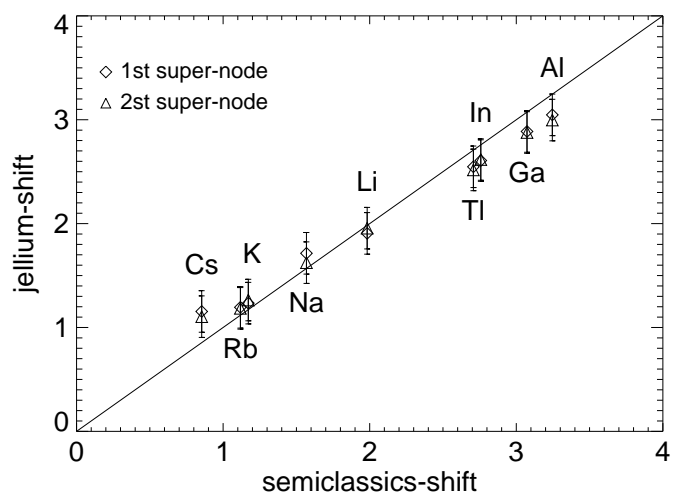

FIG. 3. Shift of super-nodes (in units of $N^{1 / 3}$ ) for different jellium clusters. The shift obtained from the periodic orbit expansion is compared to that observed in the self-consistent calculations. If these shifts agreed perfectly, the points would fall on the full line.

Although we have introduced a number of approximations the agreement is remarkable. We can thus conclude that the leptodermous expansion applies to typical cluster potentials.

To summarize, we have demonstrated how the semiclassical description of a spherical cavity can be generalized to describe the electronic supershells of realistic potentials. Starting from a periodic-orbit-expansion for the oscillating part of the total energy, we have shown that $\tilde{E}(N)$ is hardly influenced by the Maslov Phases or a lattice contraction. Introducing a leptodermous expansion for the classical action we have established that a soft potential gives phase-shifts in the semiclassical expression for $\tilde{E}(N)$. We can thus understand the dependence of the supershell on the electron density, revealed by a scaling analysis of our jellium calculations.

Moreover the leptodermous expansion can be used to analyze how the supershell structure will change if the underlying model is changed. Introducing a pseudopo- tential, as e.g. in the stabilized jellium model [21], increases the spill-out of the electrons and leads to a softening of the potential at the surface of the cluster. This induces a shift of the super-nodes towards larger $N$, which can be estimated by the semiclassical technique described above.

Finally, the identification of $r_{s}$ as the typical length scale for the supershell-problem suggests a justification of the ad-hoc procedure proposed in th to improve the results of jellium calculations for gallium clusters. There it was found that the introduction of a non-homogeneous jellium background is essential for treating $G a_{N}$ clusters, while alkali clusters are well described by a homogeneous jellium. Assuming, that the typical length scale for features in the jellium is the ionic radius $r_{a t}$, while the length scale for the electrons is the Wigner-Seitz radius $r_{s}$, we find that the importance of inhomogeneities increases with the number of valence electrons $Z_{v a l} \propto\left(r_{a t} / r_{s}\right)^{3}$.

I am much indebted to O. Gunnarsson for his invaluable advice. Helpful discussions with T. P. Martin and M. Brack are gratefully acknowledged.

[1] T. P. Martin et al., Chem. Phys. Lett. 186, 53 (1991).

[2] J. Pedersen et al., Nature (London) 353, 733 (1991).

[3] C. Bréchignac et al., Phys. Rev. B 47, 2271 (1993).

[4] M. Pellarin et al., Phys. Rev. B 48, 17645 (1993).

[5] H. Nishioka, K. Hansen, and B. R. Mottelson, Phys. Rev. B 42, 9377 (1990).

[6] O. Genzken and M. Brack, Phys. Rev. Lett. 67, 3286 (1991).

[7] R. Balian and C. Bloch, Ann. Phys. (N.Y.) 69, 76 (1972).

[8] W. Ekardt, Phys. Rev. B 29, 1558 (1984).

[9] M. C. Gutzwiller, J. Math. Phys. 11, 1791 (1970).

[10] J. Lermé et al., Phys. Rev. B 48, 9028 (1993).

[11] J. Lermé et al., Phys. Rev. B 48, 12100 (1993).

[12] J. Mansikka-aho and M. Manninen, Phys. Rev. B 48, 1837 (1993).

[13] J. Lermé et al., Phys. Rev. Lett. 68, 2818 (1992).

[14] S. H. Vosko, L. Wilk, and M. Nusair, Can. J. Phys. 58, 1200 (1980).

[15] J. R. Smith, Phys. Rev. 181, 522 (1969).

[16] C. Yannouleas and U. Landman, Phys. Rev. B 48, 8376 (1993).

[17] M. V. Berry and K. E. Mount, Rep. Prog. Phys. 35, 315 (1972).

[18] B. Tatievski, P. Stampfli, and K. H. Bennemann, Z. Phys. D 31, 287 (1994).

[19] G. Apai, J. F. Hamilton, J. Stohr, and A. Thompson, Phys. Rev. Lett. 43, 165 (1979).

[20] E. Koch, to be published.

[21] J. P. Perdew, H. Q. Tran, and E. D. Smith, Phys. Rev. B 42, 11627 (1990). 